\title{
Strain-induced modifications of transport in gated graphene nanoribbons
}

\author{
Diana A. Cosma, ${ }^{1, *}$ Marcin Mucha-Kruczyński, ${ }^{2}$ Henning Schomerus, ${ }^{1}$ and Vladimir I. Fal'ko ${ }^{1}$ \\ ${ }^{1}$ Department of Physics, Lancaster University, Lancseter LA1 4YB, United Kingdom \\ ${ }^{2}$ Department of Physics, University of Bath, Claverton Down, Bath BA2 7AY, United Kingdom \\ (Received 24 September 2014; revised manuscript received 21 November 2014; published 3 December 2014)
}

\begin{abstract}
We investigate the effects of homogeneous and inhomogeneous deformations and edge disorder on the conductance of gated graphene nanoribbons. Under increasing homogeneous strain the conductance of such devices initially decreases before it acquires a resonance structure and, finally, becomes completely suppressed at higher strain. Edge disorder induces mode mixing in the contact regions, which can restore the conductance to its ballistic value. The valley-antisymmetric pseudomagnetic field induced by inhomogeneous deformations leads to the formation of additional resonance states, which originate either from the coupling into Fabry-Pérot states that extend through the system or from the formation of states that are localized near the contacts, where the pseudomagnetic field is largest. In particular, the $n=0$ pseudo-Landau level manifests itself via two groups of conductance resonances close to the charge neutrality point.
\end{abstract}

DOI: 10.1103/PhysRevB.90.245409

PACS number(s): 73.22.Pr, 62.20.-x, 71.70.Di

\section{INTRODUCTION}

Monolayer graphene [1] is a unique material capable of sustaining reversible deformations in excess of several percent [2-6]. The effects of strain in this one-atom-thick crystalline membrane $[7,8]$ attract attention due to the peculiar way in which they affect the already unusual electronic properties of this material $[1,9,10]$. Pristine graphene displays a conical dispersion (Dirac points; DPs) at the gapless edge between the valence and the conduction bands. The DPs are replicated at the inequivalent $K$ and $K^{\prime}$ corners of the hexagonal Brillouin zone (BZ), and the effect of lattice deformations on electrons is equivalent to that of an effective gauge field with the sign inverted in the opposite valleys [2,3,11-14]. Consequently, homogeneous deformations result in a small shift of the Dirac cones from the corners of the BZ [2,20], whereas inhomogeneous strain influences electron motion similarly to a valley-dependent effective pseudomagnetic field $[9,12,15-19]$. Recent scanning-tunneling experiments on graphene nanobubbles [21] revealed that even small inhomogeneous deformations can induce pseudomagnetic fields that reach values equivalent to hundreds of teslas. Such strong fields result in the localization of the electronic states and lead to the formation of a discrete Landau level (LL) spectrum with the peculiar $n=0$ LL state positioned at zero Fermi energy $\left(E_{\mathrm{F}}=0\right)$ $[12,15-17,21-23]$.

In this paper, we perform a systematic analysis of the conductance of gated armchair graphene nanoribbons (GNRs), which are subjected to both homogeneous and inhomogeneous longitudinal deformations, as well as to various types of edge disorder. Our calculations are carried out within a tight-binding model that incorporates the strained-induced modifications of the couplings [24]. The conductance is then obtained in the Landauer-Büttiker approach [25], where the transmission probabilities are obtained using the recursive Green's function technique [26,27].

Under homogeneous deformations of increasing strength, the conductance of such ribbons first decreases, then acquires

\footnotetext{
*d.cosma@lancaster.ac.uk
}

a resonant structure, and, finally, becomes completely suppressed in a large range of energies. These effects arise from a combination of a strain-induced mismatch of the Fermi surfaces in the leads and the strained regions $[19,20]$ and the finite-size quantization of the transverse momentum. We found that these transport features are washed out by single-atom edge defects, while double-atom defects (consisting of the removal of a dimer at the edge) do not alter the resonant structure significantly and can even restore the ballistic transport properties of the ribbon in the regime where the conductance is completely suppressed by the deformations in the absence of disorder.

For completeness, we compare the above-mentioned results with those obtained for suspended GNRs [6,28-32], which display inhomogeneous strain distributions [15-17]. This includes an extended discussion of additional resonances formed by LL quantization in the pseudomagnetic field, first reported in shorter form in Ref. [33]. The nature of these resonances is revealed through the local density of states (LDOS) profiles, which we calculate at the resonance energies. We found that these features can be attributed either to Fabry-Pérot-like standing waves or to resonant transmission via pseudomagnetic LL states that form in the contact regions of the GNR. The zeroth LL is identified by its sublattice polarization $[12,23]$ and is found to result in resonance states close to the charge neutrality point.

The above-mentioned results are described in detail in Sec. III. The preceding Sec. II introduces the tight-binding model for strained graphene ribbons and identifies the underlying physics of homogeneously and inhomogeneously strained armchair GNRs, while Sec. IV summarizes their consequences.

\section{MODELING OF STRAINED GRAPHENE NANORIBBONS}

\section{A. Hamiltonian}

We consider a narrow and long-strained GNR, clamped to unstrained graphitic leads and suspended over metallic contacts. The ribbon is assumed to have free-standing armchair 

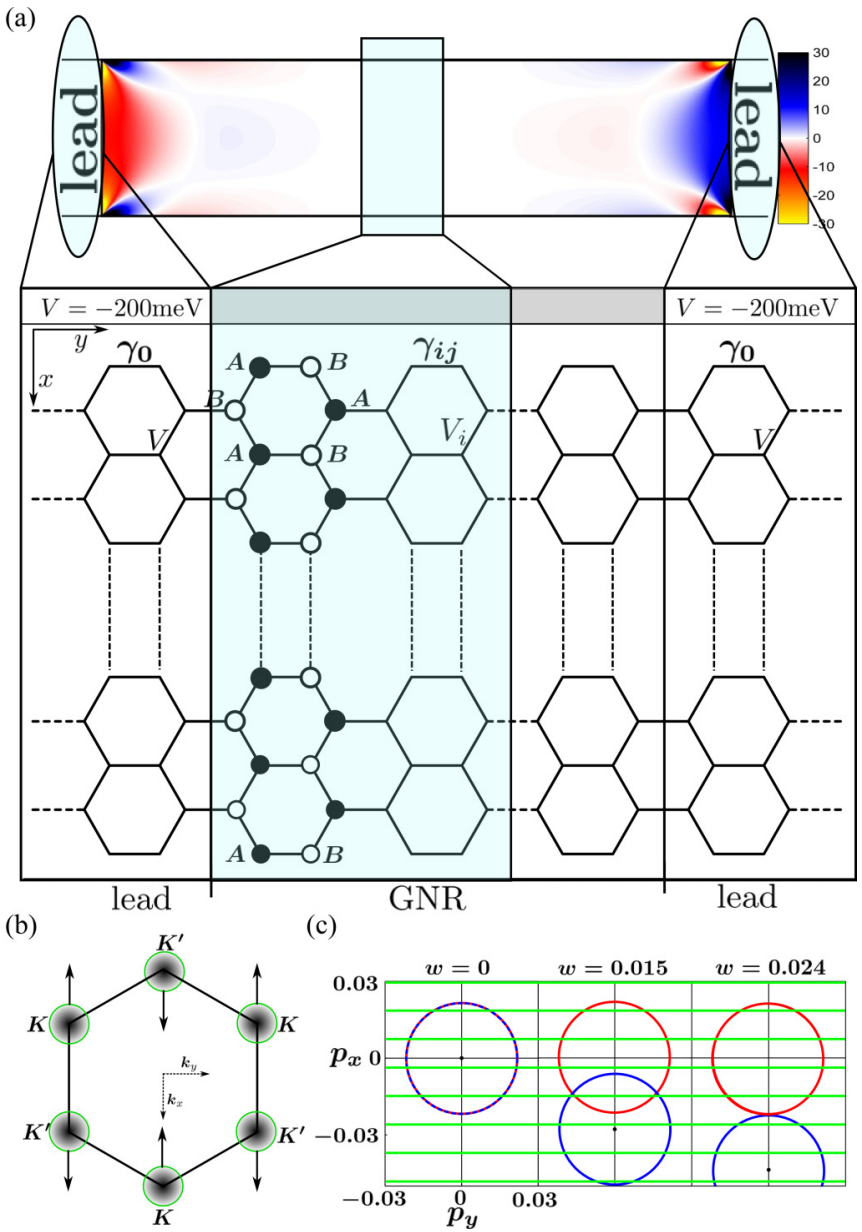

FIG. 1. (Color online) (a) Sketch of a graphene nanoribbon (GNR) of aspect ratio $L / W=4$, where $L$ is the length and $W$ is the width of the system. The color code indicates the pseudomagnetic field $\mathcal{B}$ (in teslas) for electrons in the $K$ valley; at $w=0.05$ inhomogeneous tensile strain in the middle of a system of width $W \simeq 40 \mathrm{~nm}$. We also sketch the honeycomb lattice corresponding to the tight-binding model in Eq. (1). The leads are heavily doped by imposing an on-site potential $V=-200 \mathrm{meV}$. This potential step can be controlled via electrostatic gates. In the central region the strain modulates the hopping matrix elements $\gamma_{i j}$ and the on-site energy $V_{i}$. (b) Shift of the Dirac cones from the corners $K$ and $K^{\prime}$ in the Brillouin zone of a homogeneously strained armchair GNR. (c) Fermi surfaces at $E_{\mathrm{F}}=100 \mathrm{meV}$ in the vicinity of a $K$ point in the BZ of the GNR shown in (a). We contrast the situation without strain $(w=0$; red circles) to externally imposed homogeneous strain $(w=0.015$ and 0.024 ; blue circles). Green lines represent the quantized transverse momentum values of the unstrained GNR.

edges along the transport direction $y$ and contacts with bulk electrodes along the $x$ axis, as sketched in Fig. 1(a). Such ribbons can be obtained by oriented growth on patterned $\mathrm{SiC}$ substrates [34], etching of graphene samples with catalytic nanoparticles [35], or use of chemical derivation [36]. Within the tight-binding model, the ribbon can be described by the Hamiltonian [1]

$$
\mathcal{H}=\sum_{i} V_{i} c_{i}^{\dagger} c_{i}+\sum_{\rangle i j\rangle} \gamma_{i j} c_{i}^{\dagger} c_{j},
$$

where $c_{i}$ is a fermionic annihilation operator acting on site $i$, while $\langle i j\rangle$ denotes pairs of nearest neighbors. In pristine, unstrained graphene with carbon-carbon bond lengths $r=$ $1.42 \AA$, the hopping matrix elements take the constant value $\gamma_{i j}=\gamma_{0} \approx-3 \mathrm{eV}$. The system can be doped via electrostatic gates, which induce a potential step of size $V$ at the contacts. We account for this effect in Eq. (1) by setting the on-site potential in the leads to $V_{i}=V$, while $V_{i}=0$ in the central region of an unstrained system. For strained monolayer membranes, both the on-site potential $V_{i}$ and the hopping matrix elements $\gamma_{i j}$ are modified by the deformation of the lattice. (Note that in our tight-binding approach the lattice itself remains unchanged and all deformations are absorbed into the modified hoppings, as recalculated from the microscopic theory. This procedure automatically isolates the physically relevant effects of the strain.) The on-site potential then acquires an additional contribution,

$$
V_{i}=\frac{1}{2} r \frac{\partial \epsilon_{c}}{\partial r} \operatorname{div} \boldsymbol{u}\left(\boldsymbol{r}_{i}\right)
$$

where $\boldsymbol{u}=\left(u_{x}, u_{y}\right)$ is the displacement field of the membrane and $\epsilon_{c}$ a characteristic energy function. This contribution vanishes for homogeneous strain and, furthermore, is typically well screened by the electrons in the flake and in the electrostatic environment [24]. We therefore focus on the hopping matrix elements, which must now be renormalized to [2]

$$
\gamma_{i j}=\gamma_{0} e^{\eta_{0}\left(l_{i j} / r-1\right)}, \quad l_{i j} \simeq r\left(1+\boldsymbol{n}_{i j} \cdot \hat{\boldsymbol{w}} \boldsymbol{n}_{i j}\right) .
$$

Here $l_{i j}$ is the strain-modified distance between lattice sites, $\eta_{0}=\frac{\partial \gamma_{0}}{\partial r} \frac{r}{\gamma_{0}} \approx-3$ relates the change of the nearest-neighbor coupling to the change of the bond length [37], $\hat{\boldsymbol{w}}$ is the $2 \times 2$ strain tensor $w_{\alpha \beta}=\frac{1}{2}\left(\partial_{\alpha} u_{\beta}+\partial_{\beta} u_{\alpha}\right)$ with $\alpha, \beta=x$ or $y$, and $\boldsymbol{n}_{i j}=(0,1),\left(\frac{\sqrt{3}}{2},-\frac{1}{2}\right)$, and $\left(-\frac{\sqrt{3}}{2},-\frac{1}{2}\right)$ are the unit vectors along the carbon-carbon bonds in the unstrained honeycomb lattice.

The strain-induced asymmetry in the hoppings between neighboring carbon sites is equivalent to the effect of a valleydependent gauge vector potential [12],

$$
e \mathcal{A}=\xi \frac{\hbar \eta_{0}}{2 r}\left(\begin{array}{c}
w_{x x}-w_{y y} \\
-2 w_{x y}
\end{array}\right),
$$

written for the states near one of the corners of the BZ, where $\xi=1(\xi=-1)$ for valleys $K\left(K^{\prime}\right)$.

\section{B. Homogeneous strain}

For an externally imposed homogeneous deformation, where the GNR is elongated along the $y$ axis, the elements of the strain tensor are $w_{x x}=-\sigma w, w_{y y}=w$, and $w_{x y}=0$, where $\sigma=0.165$ is the Poisson ratio for graphite [38] and $w$ parameterizes tensile strain. In this case, both the scalar and the vector potentials $V_{i}$ and $\mathcal{A}$ are constant. The scalar potential merely introduces a shift of the energy scale, which cannot be distinguished from the effect of electrostatic gating. The vector potential shifts the nonequivalent Dirac cones from the $K$ and $K^{\prime}$ corners of the BZ into opposite directions [12,20], as shown in Fig. 1(b). (Note that higher order effects induce a distortion of the Dirac cone $[39,40]$ in addition to the shift, which is neglected in this work.) Infinitely wide samples are robust 
against such deformations and their spectrum remains gapless for strains below $20 \%$ [2]. In contrast, GNRs behave markedly differently due to quantum confinement effects, which allow for an opening of the gap even for small strains $(w \ll 20 \%)$ [41-43].

Figure 1(c) shows a comparison between the Fermi surfaces around a $K$ point in the BZ, for an unstrained ribbon $(w=0$; red circles) and homogeneously strained ribbons ( $w=0.015$ and 0.024 ; blue circles) of width $W \simeq 40 \mathrm{~nm}$, at energy $E_{\mathrm{F}}=100 \mathrm{meV}$ from the DP. When the strain is smoothly increased from $w=0$ to $w=0.024$, the DP (filled black circle) crosses several quantized momentum lines (green lines) and the system undergoes multiple semiconducting-metallicsemiconducting phase transitions. Therefore, the size of the gap in the spectrum of armchair GNRs is controllable by the amount of deformation $[41,42]$, within a range determined by the width of the ribbon.

\section{Inhomogeneous strain}

To model a more realistic deformation, we assume that a suspended ribbon is clamped at the leads and stretched along the $y$ axis. Because of the clamping, the resulting deformation is inhomogeneous [44]. We neglect spontaneous wrinkling of the ribbon $[45,46]$ and consider this simplified problem within two-dimensional linear elasticity theory [47]. With the origin of the coordinate system chosen in the center of the ribbon, the displacement is then prescribed by two equations [24],

$$
\begin{aligned}
& 2 \partial_{x x} u_{x}+(1-\sigma) \partial_{y y} u_{x}+(1+\sigma) \partial_{x y} u_{y}=0, \\
& 2 \partial_{y y} u_{y}+(1-\sigma) \partial_{x x} u_{y}+(1+\sigma) \partial_{x y} u_{x}=0,
\end{aligned}
$$

accompanied by clamped boundary conditions for the left and right edge as well as free boundary conditions for the top and bottom edge:

$$
\begin{gathered}
\text { clamped }\left\{\begin{array}{l}
u_{x}(x, \pm L / 2)=0, \\
u_{y}(x, \pm L / 2)= \pm \frac{1}{2} w L,
\end{array}\right. \\
\text { free }\left\{\begin{array}{l}
{\left[\partial_{x} u_{x}+\sigma \partial_{y} u_{y}\right]_{x= \pm \frac{W}{2}}=0,} \\
{\left[\partial_{x} u_{y}+\partial_{y} u_{x}\right]_{x= \pm \frac{W}{2}}=0 .}
\end{array}\right.
\end{gathered}
$$

Despite its simplicity, the problem of finding the displacement field satisfying Eqs. (5) and (6) does not have an analytic solution, so that we apply the finite-element method [48] with a nine-point element to determine $\boldsymbol{u}(x, y)$. Having obtained the displacement [45], we calculate numerically the vector potential $\mathcal{A}(x, y)$ as predicted by the continuum model, Eq. (4). The corresponding pseudomagnetic field $\mathcal{B}(x, y)=\operatorname{rot} \mathcal{A}(x, y)$ in the $K$ valley of a GNR with width $W \simeq 40 \mathrm{~nm}$, aspect ratio $L / W=4$, and inhomogeneous tensile strain $w=0.05$ in the central part is illustrated in Fig. 1(a). The pseudomagnetic field is the largest positive (blue area) or negative (red area) near the contacts at the right and left ends and is small in the middle part of the ribbon, where the strain is approximately homogeneous. This is in contrast to the system considered in Ref. [17], where the flake is overlayed on top of a ridge and high pseudomagnetic fields develop in the central regions.

Such strong pseudomagnetic fields can lead to the quantization of electronic states into LLs and the appearance of gaps in the electronic spectrum $[12,21,24]$. Furthermore, these fields should be capable of deflecting the electrons into states that are inaccessible at homogeneous strain. In the following section, we explore these effects via the transport properties of the GNR.

\section{CONDUCTANCE}

Having established the effects of both homogeneous and inhomogeneous strains on the electronic structure of the GNRs, we now turn to the main goal of this paper and discuss the conductance of the two-terminal device sketched in Fig. 1. In our numerical procedure, we first map the displacement directly onto the crystalline lattice of the ribbon and calculate the positions of the carbon atoms after the deformation. We then recalculate the nearest-neighbor couplings according to Eq. (3) and use this information as input for the tight-binding Hamiltonian, (1). As mentioned above, we ignore the on-site scalar potential $V_{i}$, as it is screened by the electrons in the flake and the electrostatic environment [24].

The phase-coherent transport properties of such twoterminal devices are encoded in the scattering matrix [49-51],

$$
S=\left(\begin{array}{ll}
r & t^{\prime} \\
t & r^{\prime}
\end{array}\right)
$$

which we evaluate using the recursive Green's function technique [26,27] applied to the tight-binding model. Here, $t, t^{\prime}\left(r, r^{\prime}\right)$ are the transmission (reflection) amplitudes of charge carriers incident from the source or the drain leads, respectively. Using the Landauer-Büttiker formalism [25], we calculate the conductance at zero temperature,

$$
G\left(E_{\mathrm{F}}, T=0\right)=\frac{2 e^{2}}{h} \operatorname{Tr}\left(t^{\dagger} t\right)
$$

as a function of the Fermi level $E_{\mathrm{F}}$. We also consider the effects of finite temperatures, where

$$
G(\mu, T)=\frac{2 e^{2}}{h} \int d E_{\mathrm{F}}\left(-\frac{\partial f\left(E_{\mathrm{F}}-\mu\right)}{\partial E_{\mathrm{F}}}\right) \operatorname{Tr}\left(t^{\dagger} t\right) .
$$

Here $\mu$ is the chemical potential, which enters together with the temperature into the Fermi distribution $f(\varepsilon)=(1+$ $\left.\exp \left(\varepsilon / k_{\mathrm{B}} T\right)\right)^{-1}$.

Throughout the following, we set the height of the gatecontrolled potential-energy step between the doped graphene leads and the suspended part to $V=-200 \mathrm{meV}$. The resulting device is a $p$ - $p^{\prime}-p$ junction $\left(E_{\mathrm{F}}<-200 \mathrm{meV}\right)$, an $n-p-n$ junction $\left(-200<E_{\mathrm{F}}<0 \mathrm{meV}\right)$, or an $n-n^{\prime}-n$ junction $\left(E_{\mathrm{F}}>\right.$ $0 \mathrm{meV}$ ). In such systems, most of the conductance features are determined by scattering from the strain-modified $p-p^{\prime}$, $n-p$, or $n-n^{\prime}$ interfaces, a behavior which can be investigated by analyzing the spatial distribution of the electronic states. Within the used formalism, this can be revealed via the LDOS [52],

$$
\operatorname{LDOS}=\frac{i}{4 \pi} \operatorname{Tr}\left(S^{\dagger} \frac{\partial S}{\partial V_{i}}-\frac{\partial S^{\dagger}}{\partial V_{i}} S\right),
$$

which corresponds to the response of the scattering amplitudes to a small local perturbation $\delta V_{i}$ added to the Hamiltonian in Eq. (1). 

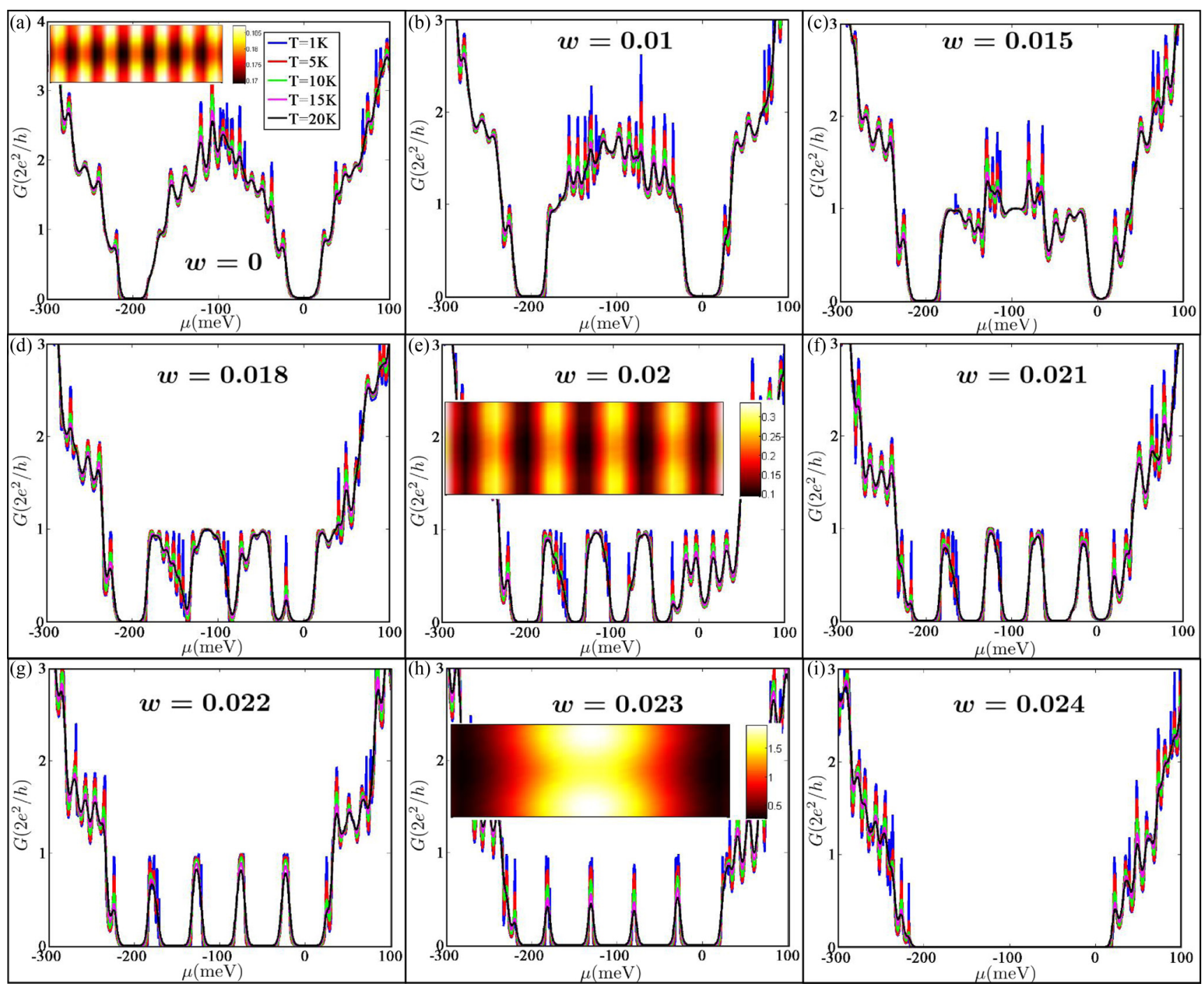

FIG. 2. (Color online) (a)-(i) Linear-response conductance as a function of chemical potential $\mu$ at several fixed temperatures $T$, for a homogeneously strained GNR of width $W \simeq 40 \mathrm{~nm}$ and aspect ratio $L / W=3$. The leads are doped via an on-site potential $V=-200 \mathrm{meV}$. Each panel corresponds to a different value of externally imposed homogeneous strain. (a), (e), (h) Spatial structure of electron wave amplitudes at energy $E_{\mathrm{F}}=-129.2 \mathrm{meV}$, evaluated using Eq. (10).

\section{A. Transport across homogeneously strained armchair GNRs}

Figure 2 shows the numerically evaluated conductance, Eq. (9), for a GNR of width $W \simeq 40 \mathrm{~nm}$ and aspect ratio $L / W=3$, as a function of chemical potential, for various values of homogeneous strain and temperature. The unstrained GNR [Fig. 2(a)] is semiconducting with a gap of $\simeq 30 \mathrm{meV}$, as determined by the quantization of the transverse momentum discussed above. The conductance exhibits two minima, at $\mu=-200$ and $0 \mathrm{meV}$, and a local maximum at $-100 \mathrm{meV}$. The conductance oscillations away from the two DPs are due to the Fabry-Pérot-like standing-wave resonances in the electron transmission across the potential barrier $[19,53,54]$. This can be seen in the LDOS profile shown in the inset, which we calculated using Eq. (10) at the resonance energy $E_{\mathrm{F}}=-129.2 \mathrm{meV}$.

For the homogeneously strained GNRs in Figs. 2(b)-2(i), the results show that the conductance continues to exhibit the minima at $\mu=-200$ and $0 \mathrm{meV}$. The effect of the strain is most noticeable in the energy range $-200 \mathrm{meV}<\mu<0 \mathrm{meV}$, where the system constitutes an $n-p-n$ junction.

For deformations $w<0.015$ [Figs. 2(b) and 2(c)], the conductance in this range is broadly suppressed. This can be attributed to the strain-induced shift of the DP, which results in a misalignment between the Fermi surfaces in the unstrained leads and the strained central region, as illustrated by the example in Fig. 1(c). Only the quantized momenta that cross the overlapping area of the two Fermi surfaces correspond to propagating modes in the leads that couple to propagating modes in the suspended region and therefore contribute to transport. With increasing strain, the area of the overlap decreases, and the conductance is reduced as an increasing number of conducting channels become blocked.

For strain $0.018 \leqslant w<0.024$ [Figs. 2(d)-2(h)] the conductance exhibits a series of well-defined resonances. In this range of strain, the area of the overlap between the Fermi surfaces in the leads and in the central region is narrower than the separation between neighboring quantized 
momenta lines. For a fixed strain $w$, the width of the overlap remains constant with varying energy, but the overlap itself is shifted in the momentum plane along the $k_{x}$ axis. Therefore, zero-conductance plateaus appear periodically in the range of energies when there is no quantized-momentum line crossing the area of the overlap. In this case, the propagating modes in the central device only couple to evanescent modes in the leads, leading to the formation of transport gaps in the system. The finite-conductance resonances are entirely due to Fabry-Pérot-like standing wave patterns, as illustrated by the LDOS profiles in Figs. 2(e) and 2(h).

For strains $w \geqslant 0.024$ [Fig. 2(i)], the conductance in the range $-200 \mathrm{meV}<\mu<0 \mathrm{meV}$ is completely suppressed, which results from the complete misalignment between the Fermi surfaces in the two regions at such strong deformations [19]. This threshold for the insulating behavior is controlled by the parameters used in Fig. 2 and can be lowered (raised) by reducing (increasing) the height of the potential step $V$ between the central part of the ribbon and the contacts.

The finite-conductance resonances are characteristic for junctions between regions of different polarity ( $n-p-n$ junctions) and are absent in junctions between regions of the same polarity ( $n-n^{\prime}-n$ and $p-p^{\prime}-p$ junctions). This is because for $\mu<-200 \mathrm{meV}$ and $\mu>0 \mathrm{meV}$ the region of overlap of the Fermi surfaces increases with increasing energy and contains an increasing number of quantized momentum lines. With larger strains $(w>0.03)$ the two Fermi surfaces will only start overlapping at energies farther away from the DPs $\left(E_{\mathrm{F}}<\right.$ $-200 \mathrm{meV}$ or $E_{\mathrm{F}}>0 \mathrm{meV}$ ), which results in a widening of the transport gap in Fig. 2(i). For example, at $w=0.05$ we find that the conductance $G$ vanishes in the entire energy range $\left|E_{\mathrm{F}}\right| \leqslant 100 \mathrm{meV}$ around the DP of the suspended region.

\section{B. Influence of edge disorder in GNRs}

Ideal ribbons with perfectly cut edges are not realistic, as most fabricated structures present a certain degree of roughness at the edges [36,55-58]. Therefore, in this subsection we establish the robustness of the strain-induced conductance resonances against edge defects. We introduce edge disorder by randomly removing a fraction $f$ of individual atoms within a strip of width $2 r$ from the edges in the strained region (single-atom vacancies) [26,59-61] and compare this to the removal of carbon-carbon dimers in the outermost rows of the edges (double-atom vacancies) [59,62]. The missing atoms are modeled by setting all the nearest-neighbor hopping elements $\gamma_{i j}$ to 0 .

Figure 3 shows the effect on the conductance at a fixed temperature $T=20 \mathrm{~K}$ for the homogeneously strained GNR of width $W \simeq 40 \mathrm{~nm}$ and aspect ratio $L / W=3$, for several strains $w$ and percentages $f$ of single-atom and double-atom vacancies as indicated in each panel. Figure 3(a) shows that single-atom defects induce smearing and suppression of the finite-conductance resonances. Previous studies have shown that in the absence of strain, such edge disorder gives rise to drastic changes in the transport properties of armchair GNRs, by inducing large fluctuations in the conductance even for small percentages of defects. By breaking the sublattice symmetry [59] and acting as short-range scatterers [60,61], such edge defects induce backscattering, Anderson-type
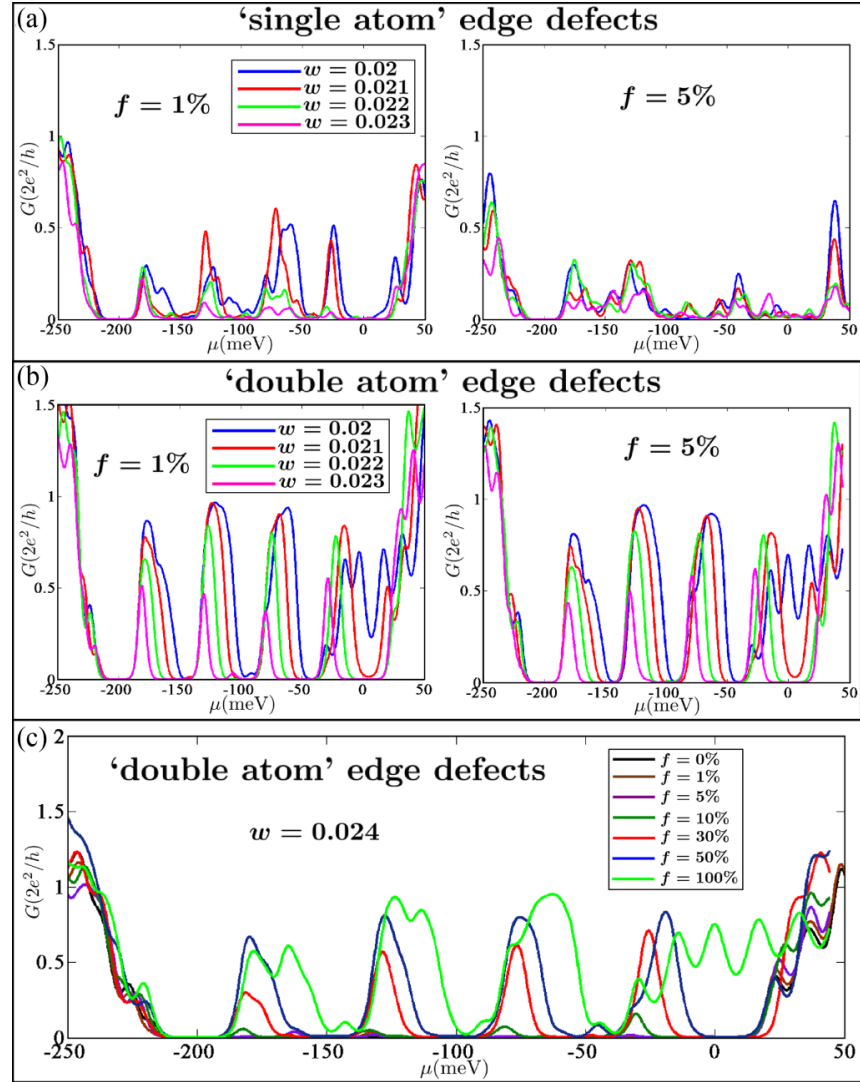

FIG. 3. (Color online) Linear-response conductance $G$ as a function of chemical potential $\mu$ at a fixed temperature $T=20 \mathrm{~K}$, for homogeneously strained GNRs, of width $W=40 \mathrm{~nm}$ and aspect ratio $L / W=3$, subjected to various types of disorder. (a) Effect of $f=1 \%$ and $5 \%$ single-atom vacancies for strain $w=0.02,0.021$, 0.022 , and 0.023 ; (b) corresponding effect of double-atom vacancies. (c) Conductance for various fractions of double-atom vacancies for a fixed strain $w=0.024$.

localization, and even the formation of conduction gaps. Similarly, our calculations show that the conductance rapidly degrades with increasing edge disorder, as an increasing number of conductive paths become blocked. Compared to the results for a defect-free system [Figs. 2(d)-2(h)], the conductance is already greatly reduced in the presence of $f=1 \%$ edge disorder, and the resonances become barely visible when $f=5 \%$.

Double-atom edge defects, on the other hand, preserve the sublattice symmetry and therefore are expected to induce only small changes in the conductance [59]. This is confirmed by the results in Fig. 3(b). Compared to the defect-free ribbon [Figs. 2(d) $-2(\mathrm{~h})]$, the conductance for $f=1 \%$ and $f=5 \%$ disorder shows remarkably little changes. Even at higher degrees of disorder, the resonances are still visible. The most significant effect is obtained for $w=0.024$ strain, depicted in Fig. 3(c), where we show the conductance calculated for various percentages of edge disorder. In this case, the transport properties of the device observed in the ballistic regime are restored by large percentages of double-atom edge defects. This behavior can be understood by comparing the two theoretical disorder extremes: $f=0 \%$ and $f=100 \%$. At $f=0 \%$ (no edge disorder), the central device and leads are perfectly matched, both having width $W$ and the same transverse momentum 
(a)
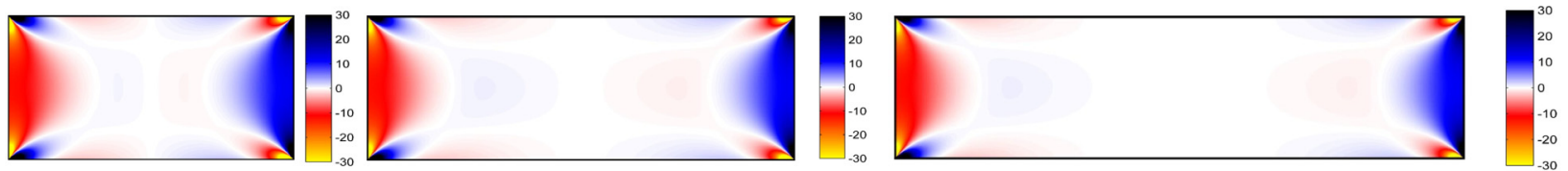

(b)
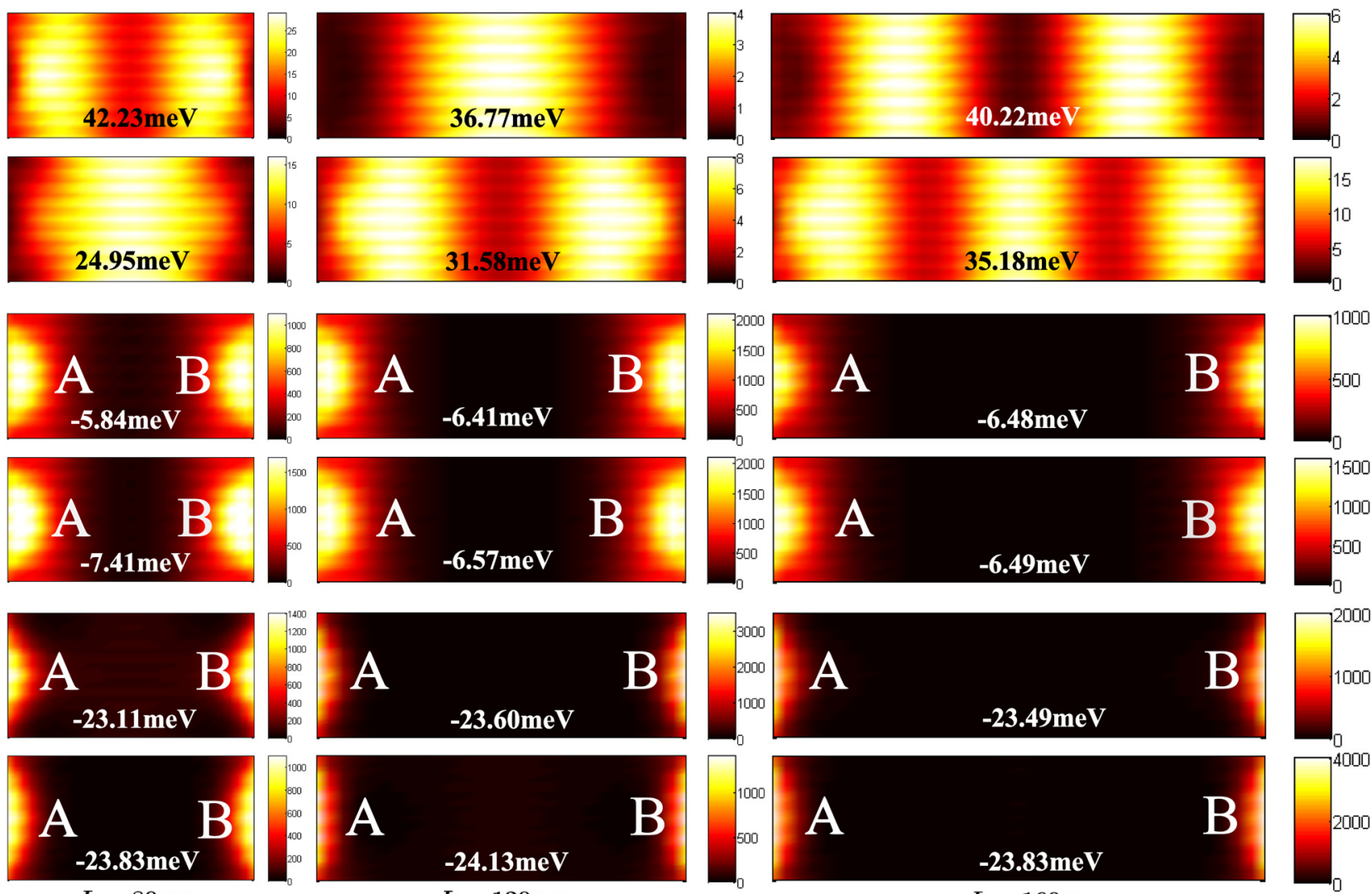

$L=80 \mathrm{~nm}$

$L=120 \mathrm{~nm}$

$L=160 \mathrm{~nm}$

FIG. 4. (Color online) (a) Suspended GNRs of width $W \simeq 40 \mathrm{~nm}$ and aspect ratios $L / W=2,3$, and 4 , which are clamped at the highly doped contacts. The color code shows the strength of the pseudomagnetic fields $\mathcal{B}$ (in teslas) for electrons in the $K$ valley, for inhomogeneous strain $w=0.05$. (b) Spatial structure of resonance states for selected resonances identified in Fig. 5.

quantization. The requirement for conservation of transverse momenta leads to the complete suppression of the conductance since the Fermi surfaces in the leads and the strained suspended region do not overlap. At $f=100 \%$ edge disorder the outermost rows of dimer lines at the top and bottom edges of the suspended region are completely removed. Therefore this region has a smaller width and correspondingly different quantized transverse momenta than the leads. This mismatch induces a mode mixing mechanism at the interfaces with the contacts, leading to the appearance of finite-conductance resonances even if the Fermi surfaces do not overlap. Other degrees of edge disorder will induce a random mixture of local boundary conditions at the edges [62] and therefore yield intermediate conductance results. Similar results were obtained by Ref. [20], where, using the continuum model, the authors showed that residual disorder restores a small finite conductivity.

\section{Transport across inhomogeneously strained armchair GNRs}

We now study the transport in suspended GNRs, which display inhomogeneous strain distributions. In contrast to clean and disordered homogeneously strained GNRs, where the conductance vanishes around the neutrality point of the suspended part, we now find that the conductance features several additional resonances, including resonances close to the neutrality point [33]. Since previous works predict the formation of pseudomagnetic LLs in such systems [12,21,24], we aim to determine whether any of the observed new features in the conductance reflect this quantization of the electronic states, without the addition of an external magnetic field as used in Ref. [17]. We focus our study on the energy range $\left|E_{\mathrm{F}}\right|<100 \mathrm{meV}$ around the DP in the suspended region, where, if present, the first few LLs are well resolved. Outside of this energy range, the states are likely to be broadened and smeared [12].

We consider three inhomogeneously strained ribbons, of width $W \simeq 40 \mathrm{~nm}$ and aspect ratios $L / W=2,3$, and 4 . The pseudomagnetic field distributions for inhomogeneous tensile strain $w=0.05$ are shown in Fig. 4(a). Using Eq. (8), we calculate the zero-temperature conductance, which is shown in the left panels in Fig. 5. In contrast to the results obtained in the previous subsections, where the conductance was completely suppressed for homogeneous strains $w \geqslant 0.024$, here we find four groups of sharp and clearly defined resonance conductance peaks for each considered aspect ratio. The two groups positioned farthest from the DP, at $E_{\mathrm{F}} \simeq-70$ and $\simeq 40 \mathrm{meV}$, contain several resonances, with their number being 


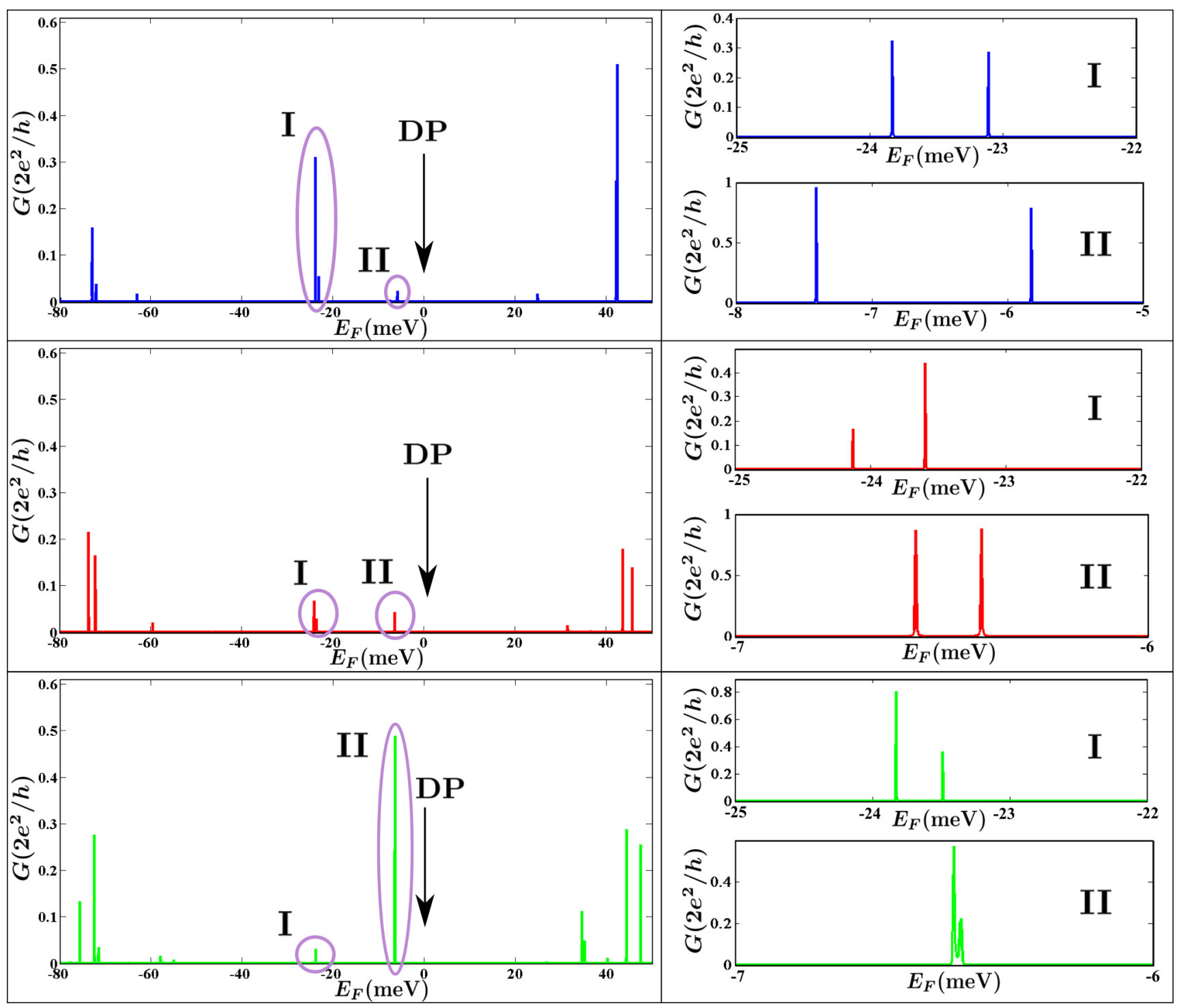

FIG. 5. (Color online) Left: Zero-temperature conductance $G$ as a function of Fermi energy for the GNRs shown in Fig. 4 . Top, $L / W=2$; middle, $L / W=3$; bottom, $L / W=4$. Right: Highly resolved results for the groups of peaks identified in the panels at the left.

proportional to the aspect ratio of the respective ribbons. For the other two groups, positioned in the energy range $-25 \mathrm{meV}<\mathrm{E}_{\mathrm{F}}<0 \mathrm{meV}$ just below the DP, the highly resolved conductance results in the right panels in Fig. 5 reveal that these resonances always occur in pairs of two. Furthermore, the splitting of the two peaks in each group decreases with increasing aspect ratio.

To uncover the origin of each group of peaks, we analyze the spatial distribution of the corresponding electronic states using Eq. (10) and arrive at the LDOS profiles shown in Fig. 4(b). As illustrated in the top two rows, the states away from the DP correspond to Fabry-Pérot-like standing waves that form due to multiple electron reflections from the left and right interfaces. Similarly to the LDOS profiles in Fig. 2, such states are confined to the central part of the structure, where the strain distribution is approximately homogeneous. The inhomogeneity near the contacts is still important, as it mixes states with different transverse momenta and thus allows the charge carriers to overcome the misalignment of the Fermi surfaces described in Sec. II. For the two groups in the energy range $-25 \mathrm{meV}<$ $\mathrm{E}_{\mathrm{F}}<0 \mathrm{meV}$, where the resonances occur in almost-degenerate pairs, the LDOS profiles shown in the bottom four rows in Fig. 4(b) do, however, point towards a very different behavior. Unlike any of the resonances we have found up to now, the spatial structure of these states clearly resembles the pseudomagnetic field distributions, which is an indicator of the formation of LLs.

As demonstrated next, this quadruplet of resonances (two groups, each containing two conductance peaks) can be attributed to the $n=0$ pseudomagnetic LL induced by the inhomogeneity at the interfaces. We exploit a unique feature of this LL in armchair GNRs, namely, that the electron amplitude resides on either the $A$ or the $B$ sublattice [12,15-17,22,23,63]. This sublattice polarization can be seen from the low-energy Hamiltonian [1],

$$
H=v_{\mathrm{F}}\left(\begin{array}{cc}
0 & \hat{\boldsymbol{\pi}}^{\dagger} \\
\hat{\boldsymbol{\pi}} & 0
\end{array}\right), \quad \begin{aligned}
& \hat{\boldsymbol{\pi}}=\hat{\boldsymbol{p}}+\frac{e}{c} \mathcal{A}, \\
& \hat{\boldsymbol{p}}=p_{x}+i p_{y} .
\end{aligned}
$$



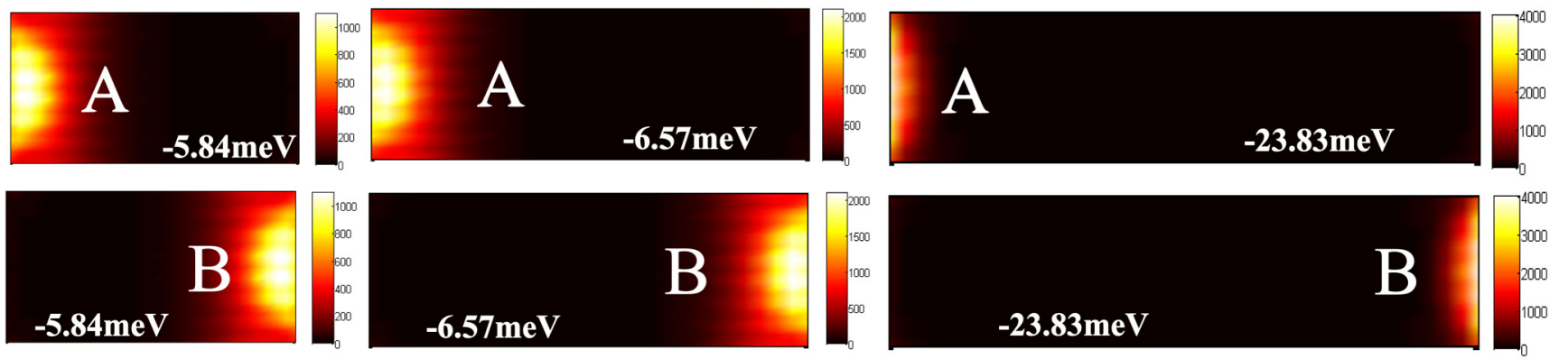

FIG. 6. (Color online) Sublattice-resolved electron amplitude for the resonances in Fig. $5\left(L / W=2,3\right.$, and 4 , at energies $E_{\mathrm{F}}=-5.84$, -6.57 , and $-23.83 \mathrm{meV}$ respectively), obtained from Eq. (10) by placing the probing perturbation $\delta V_{i}$ onto $A$ sites (top) or onto $B$ sites (bottom).

Here $v_{\mathrm{F}}$ is the Fermi velocity, $\hat{\boldsymbol{p}}$ parameterizes the in-plane momentum relative to the $K$ or $K^{\prime}$ point, and $\mathcal{A}$ is the vector potential in Eq. (4). The operator $\hat{\boldsymbol{\pi}}$ fulfills $\left[\hat{\boldsymbol{\pi}}, \hat{\boldsymbol{\pi}}^{\dagger}\right]=$ const and acts as an annihilation operator if the pseudomagnetic field is positive. Acting by this Hamiltonian on the state

$$
\left(\begin{array}{c}
|0\rangle \\
0
\end{array}\right)
$$

where $\hat{\boldsymbol{\pi}}|0\rangle=0$, we obtain the eigenvalue $E=0$. This eigenstate has a finite amplitude on the $A$ sublattice but a vanishing amplitude on the $B$ sublattice. For a negative value of the pseudomagnetic field the sublattice polarization moves onto the $B$ sublattice. However, in all cases the selected sublattice is independent of the valley [12]. In contrast, higher order LLs and Fabry-Pérot-like resonances occupy both sublattices equally [63].

By placing the probing perturbation $\delta V_{i}$ in Eq. (10) on either the $A$ or the $B$ sites, we find that the low-energy resonances are localized on the $A$ sites near the left interface (where $\mathcal{B}<0$ ) and on the $B$ sites near the right interface (where $\mathcal{B}>0$ ). This is illustrated in Fig. 6.

Further evidence that supports our interpretation of the origin of these states is the fact that we find four such low-energy resonances, with the separation between each pair inversely proportional to the aspect ratio of the ribbon. The $x \rightarrow-x$ reflection symmetry of the system maps the $K$ and $K^{\prime}$ valleys onto each other, which results in the formation of a symmetric and an antisymmetric superposition of the two valley manifestations of the $n=0 \mathrm{LL}$. This leads to a splitting of the $n=0$ LL into two branches, corresponding to each of the two groups of resonances. The branch located at $E_{\mathrm{F}} \approx-24 \mathrm{meV}$ is valley symmetric and displays a maximum on the symmetry axis. The branch located at $E_{\mathrm{F}} \approx-7 \mathrm{meV}$ is valley antisymmetric and displays a nodal line on the symmetry axis. Two states appear in each of the branches due to the hybridization of states localized at the two contacts. The tunnel coupling of these states is provided by the evanescent tails of the electronic wave functions in the central part of the system where $\mathcal{B}$ is small. Naturally, since the overlap of the evanescent tails decreases with increasing aspect ratio, the splitting in each of these pairs is smaller in a longer ribbon, thus explaining the trend we highlighted in our discussion of the highly resolved conductance result in the right panels in Fig. 5.

\section{CONCLUSION}

In conclusion, we performed a systematic study of the transport characteristics of homogeneously and inhomogeneously strained suspended armchair GNRs. The combination of strain-induced shifts of the DP in the momentum plane and size-confinement effects leads to significant modifications in the transport of homogeneously strained systems. In particular, an uncommon resonance structure appears when both of these effects compete. Large percentages of single-atom vacancies destroy the observed resonant structure. In contrast, "doublesite" vacancies do not suppress the conductance and can even restore the ballistic transport properties. For inhomogeneous deformations, we have found that the inhomogeneity developed near the contacts aids the resonant transmission of charge carriers, either through a mode mixing mechanism or through tunneling via the sublattice-polarized $n=0$ pseudomagnetic LL. The mode mixing leads to the coupling to Fabry-Pérot-like standing waves in the central part of the ribbon, which results in the formation of additional conductance peaks far from the DP. The states associated with the $n=0$ pseudomagnetic LL form near the contact regions and give rise to two pairs of conductance peaks near the DP.

\section{ACKNOWLEDGMENTS}

We thank F. Guinea and H. Ochoa for useful discussions. This project was funded by the European Graphene Flagship Project, an EPSRC S\&IA grant, ERC Synergy Grant "Hetero2D," and the Royal Society Wolfson Research Merit Award.
[1] A. H. Castro-Neto, F. Guinea, N. M. R. Peres, K. S. Novoselov, and A. K. Geim, Rev. Mod. Phys. 81, 109 (2009).

[2] V. M. Pereira, A. H. Castro Neto, and N. M. R. Peres, Phys. Rev. B 80, 045401 (2009).
[3] R. M. Ribeiro, V. M. Pereira, N. M. R. Peres, P. R. Briddon, and A. H. Castro Neto, New J. Phys. 11, 115002 (2009).

[4] F. Liu, P. Ming, and J. Li, Phys. Rev. B 76, 064120 (2007). 
[5] K. S. Kim, Y. Zhao, H. Jang, S. Y. Lee, J. M. Kim, K. S. Kim, J.-H. Ahn, P. Kim, J.-Y. Choi, and B. H. Hong, Nature 457, 706 (2009).

[6] C. Lee, X. Wei, J. W. Kysar, and J. Hone, Science 321, 385 (2008).

[7] T. J. Booth, P. Blake, R. R. Nair, D. Jiang, E. W. Hill, U. Bangert, A. Bleloch, M. Gass, K. S. Novoselov, M. I. Katsnelson, and A. K. Geim, Nano Lett. 8, 2442 (2008).

[8] J. S. Bunch, S. S. Verbridge, J. S. Alden, A. M. van der Zande, J. M. Parpia, H. G. Craighead, and P. L. McEuen, Nano Lett. 8, 2458 (2008).

[9] Z. H. Ni, T. Yu, Y. H. Lu, Y. Y. Wang, Y. P. Feng, and Z. X. Shen, ACS Nano 2, 2301 (2008).

[10] Z. H. Ni, H. M. Wang, Y. Ma, J. Kasim, Y. H. Wu, and Z. X. Shen, ACS Nano 2, 1033 (2008).

[11] S. V. Iordanskii and A. E. Koshelev, JETP Lett. 41, 574 (1985).

[12] F. Guinea, M. I. Katsnelson, and A. K. Geim, Nat. Phys. 6, 30 (2010).

[13] M. A. H. Vozmediano, M. I. Katsnelson, and F. Guinea, Phys. Rep. 496, 109 (2010).

[14] D. Rainis, F. Taddei, M. Polini, G. León, F. Guinea, and V. I. Fal'ko, Phys. Rev. B 83, 165403 (2011).

[15] H. Suzuura and T. Ando, Phys. Rev. B 65, 235412 (2002).

[16] J. L. Mañes, Phys. Rev. B 76, 045430 (2007).

[17] E. Prada, P. San-Jose, G. León, M. M. Fogler, and F. Guinea, Phys. Rev. B 81, 161402 (2010).

[18] T. Low, F. Guinea, and M. I. Katsnelson, Phys. Rev. B 83, 195436 (2011).

[19] V. M. Pereira and A. H. Castro Neto, Phys. Rev. Lett. 103, 046801 (2009).

[20] M. M. Fogler, F. Guinea, and M. I. Katsnelson, Phys. Rev. Lett. 101, 226804 (2008)

[21] N. Levy, S. A. Burke, K. L. Meaker, M. Panlasigui, A. Zettl, F. Guinea, A. H. Castro Neto, and M. F. Crommie, Science 329, 544 (2010).

[22] H. Schomerus and N. Y. Halpern, Phys. Rev. Lett. 110, 013903 (2013).

[23] C. Poli, J. Arkinstall, and H. Schomerus, Phys. Rev. B 90, 155418 (2014).

[24] M. Mucha-Kruczynski and V. I. Fal'ko, Solid State Commun. 152, 1442 (2012).

[25] M. Büttiker, Phys. Rev. Lett. 57, 1761 (1986).

[26] J. P. Robinson and H. Schomerus, Phys. Rev. B 76, 115430 (2007).

[27] S. Datta, Electronic Transport in Mesoscopic Systems (Cambridge University Press, Cambridge, 1988).

[28] K. I. Bolotin, K. J. Sikes, Z. Jiang, M. Klima, G. Fudenberg, J. Hone, P. Kim, and H. L. Stormer, Solid State Commun. 146, 351 (2008)

[29] X. Du, I. Skachko, A. Barker, and E. Y. Andrei, Nature Nanotech. 3, 491 (2008).

[30] W. Bao, K. Myhro, Z. Zhao, Z. Chen, W. Jang, L. Jing, F. Miao, H. Zhang, C. Dames, and C. N. Lau, Nano Lett. 12, 5470 (2012).

[31] H. Zhang, J.-W. Huang, J. Velasco Jr., K. Myhro, M. Maldonado, D. D. Tran, Z. Zhao, F. Wang, Y. Lee, G. Liu, W. Bao, and C. N. Lau, Carbon 69, 336 (2014).

[32] Z. J. Qi, J. A. Rodriguez-Manzo, A. R. Botello-Méndez, S. J. Hong, E. A. Stach, Y. W. Park, J.-C. Charlier, M. Drndic, and A. T. C. Johnson, Nano Lett. 14, 4238 (2014).
[33] D. A. Gradinar, M. Mucha-Kruczynski, H. Schomerus, and V. I. Fal'ko, Phys. Rev. Lett. 110, 266801 (2013).

[34] J. Hicks, A. Tejeda, A. Taleb-Ibrahimi, M. S. Nevius, F. Wang, K. Shepperd, J. Palmer, F. Bertran, P. Le Fèvre, J. Kunc, W. A. de Heer, C. Berger, and E. H. Conrad, Nat. Phys. 9, 49 (2012).

[35] L. C. Campos, V. R. Manfrinato, J. D. Sanchez-Yamagishi, J. Kong, and P. Jarillo-Herrero, Nano Lett. 9, 2600 (2009).

[36] X. Li, X. Wang, L. Zhang, S. Lee, and H. Dai, Science 319, 1229 (2008).

[37] R. Ferone, J. R. Wallbank, V. Zolyomi, E. McCann, and V. I. Fal'ko, Solid State Commun. 151, 1071 (2011).

[38] O. L. Blakslee, D. G. Proctor, E. J. Seldin, G. B. Spence, and T. Weng, J. Appl. Phys. 41, 3373 (1970).

[39] F. de Juan, M. Sturla, and M. A. H. Vozmediano, Phys. Rev. Lett. 108, 227205 (2012).

[40] S.-M. Choi, S.-H. Jhi, and Y.-W. Son, Phys. Rev. B 81, 081407(R) (2010).

[41] Y. Li, X. Jiang, Z. Liu, and Z. Liu, Nano Res. 3, 545 (2010).

[42] Y. Lu and J. Guo, Nano Res. 3, 189 (2010).

[43] S. H. R. Sena, J. M. Pereira Jr., G. A. Farias, F. M. Peeters, and R. N. Costa Filho, J. Phys.: Condens. Matter 24, 375301 (2012).

[44] J. L. Mañes, F. de Juan, M. Sturla, and M. A. H. Vozmediano, Phys. Rev. B 88, 155405 (2013).

[45] Note that we have neglected the possibility of spontaneous wrinkling [46] since strain limits their formation in suspended samples by increasing the transverse rigidity [64]. We have also applied a finite cutoff to regularize the formally divergent displacements [65] one finds using linear elasticity theory [47] near the corners of the clamped ends of the ribbon.

[46] E. Cerda and L. Mahadevan, Phys. Rev. Lett. 90, 074302 (2003).

[47] S. P. Timoshenko and J. N. Goodier, Theory of Elasticity (McGraw-Hill, Singapore, 1970).

[48] O. C. Zienkiewicz and R. L. Taylor, The Finite Element Method, Vol. I-III, 5th ed. (Butterworth-Heinemann, Oxford, 2000).

[49] H. Schomerus, Phys. Rev. B 76, 045433 (2007).

[50] C. W. J. Beenakker, Rev. Mod. Phys. 69, 731 (1997).

[51] Y. M. Blanter and M. Büttiker, Phys. Rep. 336, 1 (2000).

[52] V. Gasparian, T. Christen, and M. Büttiker, Phys. Rev. A 54, 4022 (1996).

[53] M. I. Katsnelson, K. S. Novoselov, and A. K. Geim, Nat. Phys. 2, 620 (2006).

[54] T. Tudorovskiy, K. J. A. Reijnders, and M. I. Katsnelson, Phys. Scripta T146, 014010 (2012).

[55] M. Y. Han, B. Özyilmaz, Y. Zhang, and P. Kim, Phys. Rev, Lett. 98, 206805 (2007).

[56] L. Jiao, X. Wang, G. Diankov, H. Wang, and H. Dai, Nat. Nanotech. 5, 321 (2010).

[57] E. J. H. Lee, K. Balasubramanian, R. T. Weitz, M. Burghard, and K. Kern, Nat. Nanotech. 3, 486 (2008).

[58] A. Cresti, N. Nemec, B. Biel, G. Niebler, F. Triozon, G. Cuniberti, and S. Roche, Nano Res. 1, 361 (2010).

[59] T. C. Li and S.-P. Lu, Phys. Rev. B 77, 085408 (2008).

[60] M. Evaldsson, I. V. Zozoulenko, H. Xu, and T. Heinzel, Phys. Rev. B 78, 161407 (2008). 
[61] E. R. Mucciolo, A. H. Castro Neto, and C. H. Lewenkopf, Phys. Rev. B 79, 075407 (2009).

[62] D. A. Areshkin, D. Gunlycke, and C. T. White, Nano Lett. 7, 204 (2007).

[63] Y. Zheng and T. Ando, Phys. Rev. B 65, 245420 (2002).
[64] E. V. Castro, H. Ochoa, M. I. Katsnelson, R. V. Gorbachev, D. C. Elias, K. S. Novoselov, A. K. Geim, and F. Guinea, Phys. Rev. Lett. 105, 266601 (2010).

[65] M. L. Williams, J. Appl. Mech. Trans. ASME 19, 526 (1952). 